International Journal of Bifurcation and Chaos, Vol. 9, No. 12 (1999) 2329-2333

(C) World Scientific Publishing Company

\title{
ANOMALOUS RELATIONSHIP BETWEEN SPATIAL AND TEMPORAL PATTERNS OF DYNAMICAL BEHAVIOR
}

\author{
LEONID RUBCHINSKY* ${ }^{*}$ and MIKHAIL SUSHCHIK ${ }^{\dagger}$ \\ Institute of Applied Physics, Russian Academy of Science, \\ 46 Ulyanov st., Nizhny Novgorod, 603600, Russia
}

Received August 1, 1998, Revised February 1, 1999

\begin{abstract}
The relationship between spatial and temporal patterns of dynamical behavior is studied in the nonequilibrium, extended homogeneous system. We present an example of "anomalous" relationship between spatial and temporal patterns of behavior and discuss possible mechanisms of this phenomenon. By anomalous relationship we mean a situation when only chaotic temporal dynamics is possible in the spatially homogeneous regime, while in the spatially inhomogeneous regime temporal dynamics can be regular at the same value of parameters. We studied this phenomenon in the array of identical coupled Chua's circuits.
\end{abstract}

\section{Introduction}

Complex spatiotemporal patterns in extended systems are formed as a result of developing hierarchy of instabilities as supercriticality is increased. One can frequently distinguish two limiting cases at the initial stage of their formation (see e.g. [Rabinovich \& Sushchik, 1990]). In the first of them, an increase of supercriticality leads to complication of the spatial structure, which is not involved with complication of the temporal dynamics. For example, this is observed in convection at large Prandtl numbers. In the other case, complex temporal behavior, chaotic one included, may occur almost without changes in the regular spatial structure. An example is a cylindric Couette flow.

With a further increase of supercriticality, the changes in the spatial structure and temporal behavior become coupled with each other. Complication of the spatial structure of such a system usually leads to complication of its temporal behavior. This situation is quite plausible taking into consideration that the dimension of chaos depends on the number of collective excitations at large values of supercriticality [Aranson et al., 1985].

In this connection a question arises: "Is a reverse relationship possible, when more complicated spatial structure corresponds to more regular temporal behavior?" More specifically, we will be interested whether two alternative types of behavior may coexist in a homogeneous system at fixed parameter values, viz. a spatially homogeneous regime in which only chaotic temporal dynamics is possible, and spatially inhomogeneous regimes (irregular ones inclusive, i.e. spatially disordered regimes) when regular temporal dynamics is possible. Let us call it anomalous relationship between temporal and spatial patterns of behavior (ARP, anomalous relationship between patterns). If these alternative types of behavior really coexist, then what are the mechanisms of the formation of such an "anomalous" multistability? In this research we give positive answers to these questions and

*E-mail: leo@hale.appl.sci-nnov.ru

${ }^{\dagger}$ E-mail: sushch@euler.appl.sci-nnov.ru 
suggest one of the possible mechanisms underlying ARP.

\section{Model}

The present research is focused on the mechanism of ARP based on bistability of an isolated element of an extended system. Thus, it is convenient to consider the problems formulated in the Introduction in the arrays of coupled Chua's circuits, wellstudied systems known to possess multistability in a broad region of parameters [Madan, 1993; Chua, 1993; Mira, 1997]. This is one of the reasons why Chua's circuits are actively used in the investigations of different phenomena in multistable systems [Nekorkin \& Chua, 1993; Kazantsev et al., 1997].

The analysis presented in this paper is restricted to a particular model that can be written in the form of three ordinary differential equations:

$$
\begin{aligned}
& \dot{x}=\alpha(y-x-h(x)) \\
& \dot{y}=x-y+z \\
& \dot{z}=-\beta y .
\end{aligned}
$$

To approximate a nonlinear characteristic of the nonlinear element of the circuit we take a smooth function

$$
h(x)=c_{1} x^{3}-\frac{2 c_{0} x}{1+c_{0}^{2} x^{2}} .
$$

The bifurcations in this particular form of Chua's circuit for the values of parameters that interest us were studied in [Kuznetsov, 1998]. The phase space of Eq. (1) is symmetric with respect to $(x, y, z) \rightarrow$ $(-x,-y,-z)$ transformation.

We model a one-dimensional array of these elements by

$$
\begin{gathered}
\dot{x}_{i}=\alpha\left(y_{i}-x_{i}-h\left(x_{i}\right)\right)+d \tanh \left(\frac{x_{i-1}-x_{i}}{\sigma}\right) \\
+d \tanh \left(\frac{x_{i+1}-x_{i}}{\sigma}\right) \\
\dot{y}_{i}=x_{i}-y_{i}+z_{i} \quad i=1, \ldots, N \\
\dot{z}_{i}=-\beta y_{i}
\end{gathered}
$$

$\left(x_{0}, y_{0}, z_{0}\right)=\left(x_{1}, y_{1}, z_{1}\right)$ and $\left(x_{N}, y_{N}, z_{N}\right)=$ $\left(x_{N+1}, y_{N+1}, z_{N+1}\right)$ are the boundary conditions (an array with free ends).

The type of coupling we use corresponds to the resistive kind of coupling with saturation. We took this particular form of the element of an array and designed the type of coupling to build up a very simple and instructive example of ARP. As we will discuss below, there should be some generality of ARP, but in Eq. (2) the ARP effect is very transparent. Numerical experiments described below were performed with a chain consisting of $N=100$ circuits.

\section{Anomalous Relationship between Patterns}

Two spatially homogeneous (i.e. independent of discrete coordinate $i$ ) solutions with chaotic temporal behavior exist in the system (2) in a certain domain of parameter space (we took $\alpha=6.32$, $\beta=10, c_{0}=0.65$, and $c_{1}=0.05$; the coupling parameters were $d=0.2, \sigma=0.00001)$. In this case, $\left(x_{i}, y_{i}, x_{i}\right)=\left(x_{j}, y_{j}, z_{j}\right)$ for any pair $(i, j)$ at any time instant after transition period and $x, y$ and $z$ of any element evolve in time chaotically (see Fig. 1). This regime corresponds to the definition of chaotic synchronization given in [Afraimovich et al., 1986] and [Pecora \& Carrol, 1990] and very similar to that, studied in [Perez-Villar et al., 1993]. The value of the coupling term

$$
D=d \tanh \left(\frac{x_{i-1}-x_{i}}{\sigma}\right)+d \tanh \left(\frac{x_{i+1}-x_{i}}{\sigma}\right)
$$

is equal to zero in this case and the dynamics of any element of the chain coincides with the dynamics of the isolated circuit (1). We would like to note that two temporally chaotic solutions are the only nontrivial homogeneous solutions that exist in the system because the only attractors in Eq. (1) for our parameter values are two strange attractors (one of them transforms to the other under the change of signs of variables). Small perturbations of this regime lead to a variation of $D$ from zero that drives the system back to the state of ideal chaotic synchronization, i.e. dissipative coupling makes this regime stable.

Let us take as initial conditions an inhomogeneous spatial structure. Then, we will obtain a regime which is completely different from the chaotic synchronization described above. Temporal behavior of the elements of the chain become regular and individual Chua's circuits generate periodic oscillations (Fig. 2). Circuits that are close to each other are often mutually synchronized. If we take another inhomogeneous spatial structure 

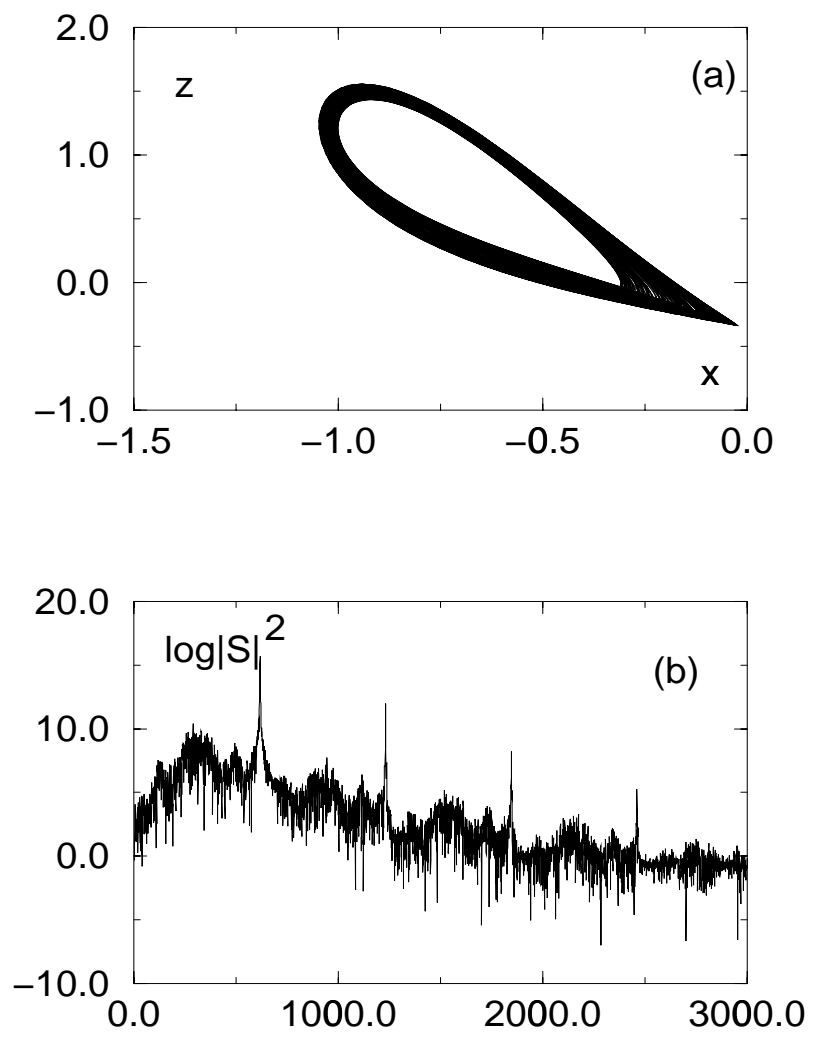

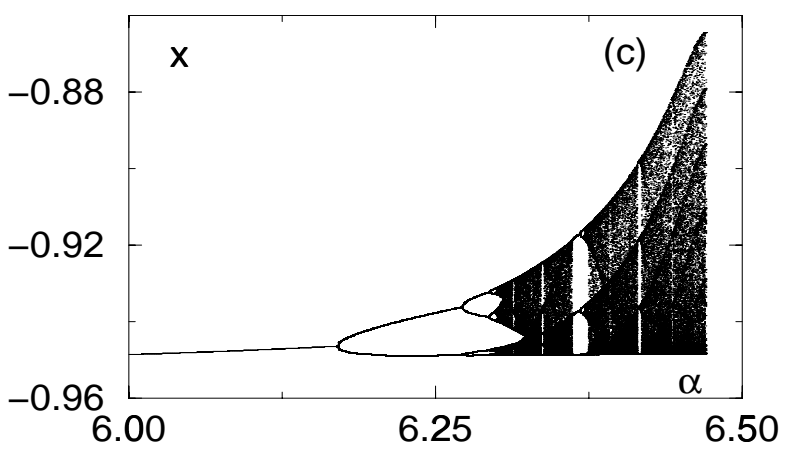

Fig. 1. (a) An attractor of isolated circuit (1); (b) power spectrum of $x(t)$ of an isolated circuit, note the continuous character of the spectrum; (c) period-doubling cascade in Eq. (1), $x$-coordinate of intersections of a trajectory with $y=0$ plane is plotted versus bifurcational parameter $\alpha$.

as the initial conditions we may get another spatial distribution with regular temporal behavior. Certain initial conditions give rise to locally inhomogeneous regimes, where some oscillators behave regularly while the other ones are chaotic.

Thus, the system (2) possesses a high degree of multistability but, in general, it is possible to distinguish two different types of behavior: two spatially homogeneous chaotic regimes
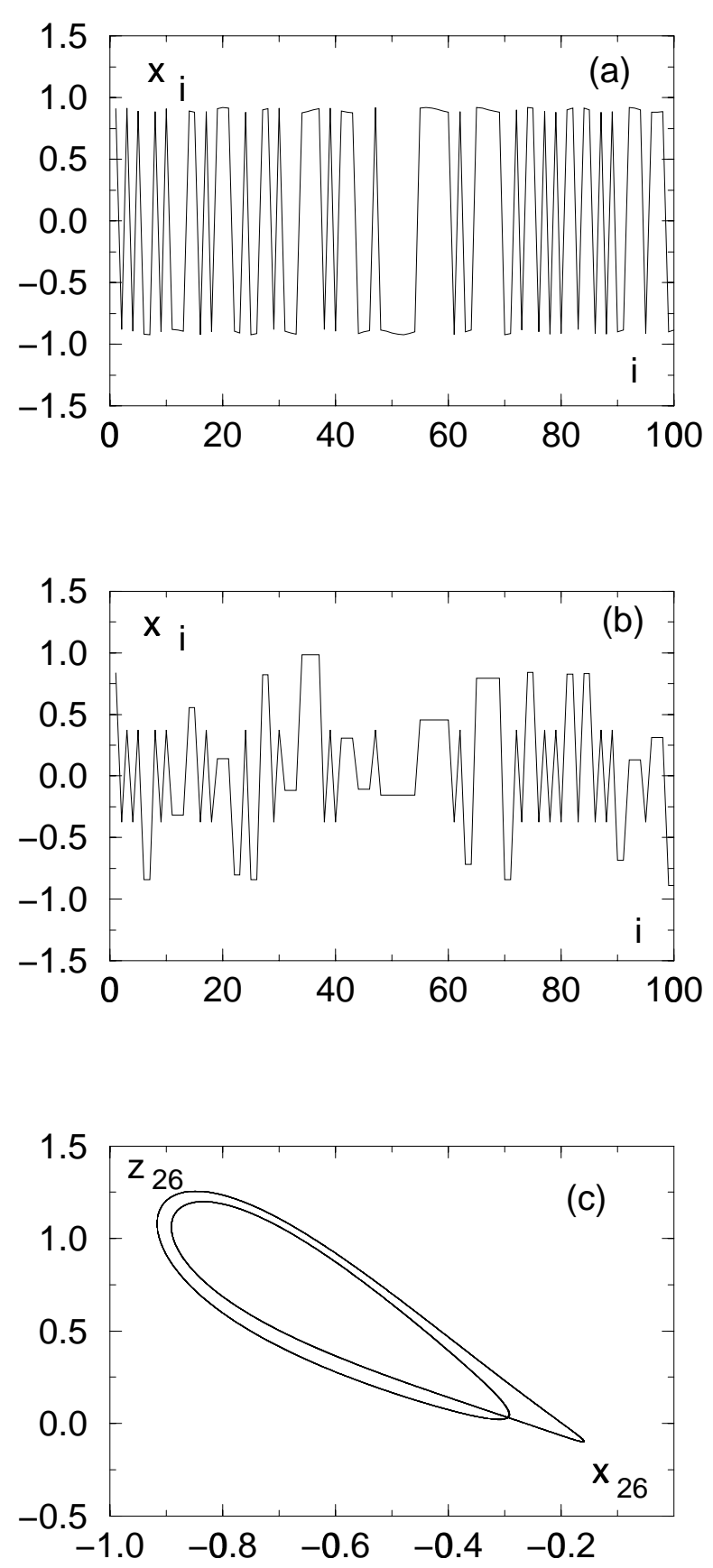

Fig. 2. Regular inhomogeneous regime. (a) Initial conditions (to make the picture clear we plotted only $x_{i}$ ), (b) snapshot of inhomogeneous spatial pattern observed after transition period, (c) projection of the attractor of (2) to $\left(x_{26}, y_{26}\right)$ plane.

and spatially inhomogeneous regular regimes. An autocorrelation function of spatial distribution from Fig. 2(b)

$$
C(j)=\left\langle\left(x_{i}-\langle x\rangle\right)\left(x_{i+j}-\langle x\rangle\right)\right\rangle,
$$

where \langle\rangle stands for discrete space average, is given in Fig. 3 in comparison with the autocorrelation 


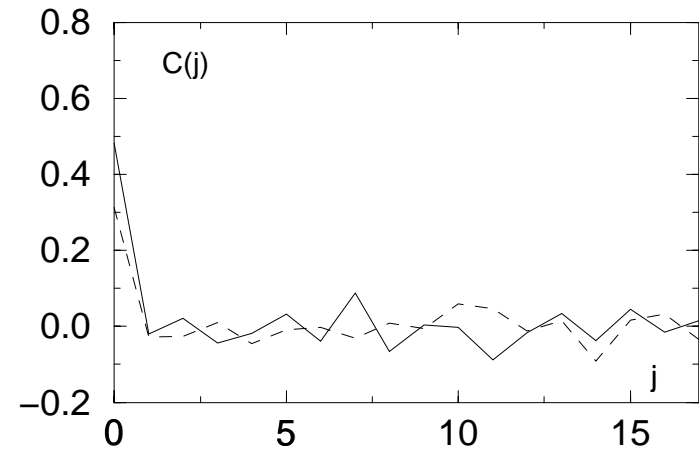

Fig. 3. Autocorrelation function $C\left(x_{i}, x_{i+j}\right)$ of the spatial distribution of the snapshot of $x_{i}$ [Fig. 2(b)] (solid line). Note that distant oscillators are spatially uncorrelated. For comparison, we plotted autocorrelation function for the sequence produced by random number generator (dashed line).

function for a sequence of random numbers obtained from the library of our $\mathrm{C}$ compiler. The absence of correlations between distant points indicates rather irregular spatial pattern.

\section{Discussion}

So far we presented an example, where we found somewhat an unexpected result: Only chaotic temporal dynamics is possible in the spatially homogeneous regime, while temporal dynamics in the spatially inhomogeneous regime can be regular at the same values of parameters. To get an insight into possible mechanism of ARP, it is very helpful to consider a degenerate case of ARP when every odd and even oscillators oscillate near $S A_{-}$and $S A_{+}$, respectively $\left(x_{2 i+1}=x_{1}, x_{2 i}=x_{2}\right){ }^{1}$

We easily observed such a regime for the specified parameter values we used to demonstrate ARP. Note that if we impose periodic boundary conditions we get a solution, which is equivalent to the corresponding solution in an array of just two coupled circuits (types of the stability of these solutions may be different). Therefore it is reasonable to suppose that the mechanism of ARP can be studied in the framework of a relatively simple system of two coupled Chua's circuits. Numerical modeling of this system showed that for " $S A_{-}$" initial conditions in one circuit and " $S A_{+}$" initial conditions in the other circuit a regularization of behavior is achieved for sufficiently large values of $d$. The influence of an oscillator on its neighbor is equivalent to a small constant added to the RHS of the equation for $\dot{x}$ in Eq. (1). This leads to the "movement" of the system in the parameter space. Depending on coupling characteristics, it may lead to other regimes (less or more chaotic [Kuznetsov, 1998]). In our case the system is displaced from the domain of chaotic behavior to the region of perioddoubling cascade. Depending on the value of $d$ we can get $1,2, \ldots, n$ periodic oscillations (for small and zero $d$, the behavior is chaotic). Analogous situation was observed in (2). We suppose that the proposed mechanism of ARP is general enough to suspect that it can be observed in some other bistable chaotic systems with period-doubling cascade and ARP is not restricted to the "artificially" designed system (2). We observed similar multistability in numerical experiments with a chain consisting of 100 van der Pol-Duffing oscillators coupled by reactive nonlinear coupling (these results will be reported elsewhere).

To conclude we would like to make two comments.

1. Our consideration is restricted to a limiting case when the regimes of chaotic synchronization are stable. The stability is attained by choosing a rather large value of the derivative of the coupling function $D$ for zero argument. As the derivative of $D$ for zero argument is decreased (or the number of oscillators is increased) the synchronization breaks down, first of all, relative to longwave perturbations, so that the spatial regularity is retained at rather long distances and the dynamics persists to be chaotic and, perhaps, even more complicated. In addition to this regime, the regimes that are strongly irregular in space and possess simpler dynamics may exist as before. Thus, the qualitative statement that APR is available in the systems of interest in the absence of stable chaotic synchronization holds true but its further description needs the introduction of special quantitative characteristics of spatiotemporal patterns, which will be considered elsewhere.

2. Temporal dynamics becomes more regular when inhomogeneous initial conditions (in particular, spatial disorder) are specified in systems with

\footnotetext{
${ }^{1}$ Let us remind that two attractors exist in the phase space of Eq. (1) in the domain of parameters that is interesting for us: strange attractors $S A_{-}$and $S A_{+}$. One of them transforms into the other under $(x, y, z) \rightarrow(-x,-y,-z)$ since the phase spaces of Eqs. (1) and (2) are symmetric. These attractors are born via period-doubling cascade with the increase of $\alpha$ [Fig. 1(c)].
} 
ARP. This resembles the effect known as taming spatiotemporal chaos with disorder [Braiman et al., 1995; Lindner et al., 1997] when the initially chaotic dynamics of arrays of oscillators becomes more regular due to spatially inhomogeneous spread of parameters of the oscillators in the array. Apparently, the system becomes inhomogeneous in this case. Contrary to the studies by Braiman et al. [1995] and Lindner et al. [1997], in the case described in the present paper, the system itself persists to be homogeneous and the spatial inhomogeneity that is the primary cause of regularization of dynamics is formed in a self-consistent fashion under the appropriate choice of initial conditions.

\section{Acknowledgments}

This work was supported by the Russian Foundation for Basic Research (project 97-02-17526) and the "Leading Scientific Schools of the Russian Federation" Program (project 96-15-96593). The authors are grateful to A. S. Kuznetsov for useful discussions. L. Rubchinsky would like to thank the organizing committee of the Workshop "Synchronization and Pattern Formation in Coupled Oscillators", in particular Dr. Vicente Perez-Munuzuri, for their hospitality and support, and many participants of the Workshop for interesting discussions. L. Rubchinsky also aknowledges the International Soros Science Educational Program grant.

\section{References}

Afraimovich, V. S., Verichev, N. N. \& Rabinovich, M. I. [1986] "Stochastic synchronization of oscillations in dissipative systems," Radiophys. Quant. Electron. 29, 795-803.

Aranson, I. S., Gaponov-Grekhov, A. V. \& Rabinovich, M. I. [1985] "The development of chaos in dynamic structure ensembles," JETP 62, 52-96.

Braiman, Y., Lindner, J. F. \& Ditto W. L. [1995] "Taming spatiotemporal chaos with disorder," Nature 378, 465-467.

Chua, L. O. [1993] "Global unfolding of Chua's circuit," IEICE Trans. Fundamental Electron. Commun. Comput. Sci. E76A5, 704-737.

Kazantsev, V. B., Nekorkin, V. I. \& Velarde, M. G. [1997] "Pulses, fronts and chaotic wave trains in a onedimensional Chua's lattice," Int. J. Bifurcation and Chaos 7, 1775-1790.

Kuznetsov, A. S. [1998] "Dynamics of modified Chua's circuit," Bulletin of Nizhny Novgorod State University. Radiophysics I, pp. 135-146 (in Russian).

Lindner, J. F., Prusha, B. S. \& Clay, K. E. [1997] "Optimal disorders for taming spatiotemporal chaos," Phys. Lett. A231, 164-172.

Madan, R. N. (ed.) [1993] Chua's Circuit: A Paradigm for Chaos (World Scientific, Singapore).

Mira, C. [1997] "Chua's circuit and the qualitative theory of dynamical systems," Int. J. Bifurcation and Chaos 7, 1911-1916.

Nekorkin, V. I. \& Chua, L. O. [1993] "Spatial disorder and wave fronts in a chain of coupled Chua's circuits," Int. J. Bifurcation and Chaos 3, 1282-1292.

Pecora, L. M. \& Carrol, T. L. [1990] "Synchronization in chaotic systems," Phys. Rev. Lett. 64, 821-824.

Perez-Villar, V., Munuzuri, A. P., Perez-Munuzuri, V. \& Chua, L. O. [1993] "Chaotic synchronization of a one-dimensional array of nonlinear active systems," Int. J. Bifurcation and Chaos 3, 1067-1074.

Rabinovich, M. I. \& Sushchik, M. M. [1990] "The regular and chaotic dynamics of structures in fluid flows," Sov. Phys. Usp. 33, 1-35. 